\title{
Das Konzept der Chronizität psychischer Erkrankungen ist aufzugeben
}

\author{
The Concept of Chronicity of Psychiatric Disorders Should be Given up
}

\section{Bibliografie}

DOI $10.1055 / \mathrm{s}-2008-1067423$

Psychiat Prax 2009; 36: 4-6

(c) Georg Thieme Verlag KG

Stuttgart · New York .

ISSN 0303-4259

Korrespondenzadressen

Univ.-Prof. Dr.

Michaela Amering

Universitätsklinik für Psychiatrie und Psychotherapie,

Abteilung für Sozialpsychiatrie

Währinger Gürtel 18-20

1090 Wien, Österreich

michaela.amering@

meduniwien.ac.at

PD Dr. med. habil. Ronald Bottlender

East London NHS Foundation

Trust, Newham Centre for

Mental Health, Glen Road,

London E13 8SP

Ronald.Bottlender@

eastlondon.nhs.uk

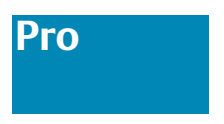

\section{Chronisch ist retrospektiv lang dauernd und prospektiv unver- änderbar}

Nils Greve zitiert Bruno Hilden-

brand [1] und erfasst damit einen Gutteil dessen, was die Sorgen darum ausmacht, ob das Modell der chronischen Erkrankung geeignet ist, Hilfeund Selbsthilfeanstrengungen optimal zu nutzen.

Leider wird Chronizität häufig so interpretiert, als würde es bedeuten auf ewig schicksalhaft einer Erkrankung und ihren unausweichlichen Folgen ausgeliefert zu sein. Ein Kreislauf aus Hoffnungs- und Tatenlosigkeit kann zu Schwächungen führen und Verschlechterungen im Gesundheitszustand nach sich ziehen bzw. zum Erhalt von Symptomen und Behinderungen beitragen. Der aus einer solchen Interpretation resultierende Mangel an Zuversicht in therapeutische Angebote kann rechtzeitige und nachhaltige Hilfesuche untergraben und die Idee der Gesundheit sowohl als Ziel als auch als Ressource in den Hintergrund drängen. Solche gesundheitsstörenden Missverständnisse entfalten ihre ungünstigen Wirkungen unabhängig davon, dass aus klinischer Sicht Chronizität einer Störung oder einer Vulnerabilität per se keineswegs im Widerspruch $\mathrm{zu}$ einem hohen Maß an Gesundheit und zu Chancen auf Genesung steht.

Wie ein „Code der Chronizität“ das Leben in einer psychiatrischen Einrichtung prägen und Anstrengungen in Richtung Besserung untergraben kann, beschrieben 1966 Ludwig und Farrelly [2] für eine Institution, in der PatientInnen genauso wie professionelle HelferInnen einem solchen Code unterliegen: Der therapeutische Eifer erlahmt. Vorherrschend ist der Wunsch nach Ordnung und das Abwehren von Veränderungen. Oberflächliche Compliance führt zu einem reduzierten Leben in einem geschützten Rahmen mit einem Minimum an Beanspruchung, Verantwor- tung und Risiko und einem Maximum an Ruhe und Vorhersehbarkeit.

Das Erkennen der negativen Folgen eines solchen Institutionalismus trug dazu bei, dass es heute zumindest in der westlichen Welt keine derartigen Großinstitutionen mehr gibt. Ob und in welchem Ausmaß Phänomene des Institutionalismus mit der Psychiatriereform in die gemeindenahe Psychiatrie mitgegangen sind, ist eine wichtige Frage. Vieles deutet darauf hin, dass auch in ambulanten Einrichtungen oder kleinen Institutionen ähnliche Phänomene auftreten können.

Vorhersagen sind schwierig, besonders wenn sie die Zukunft betreffen (Karl Valentin; Nils Bohr)

Der Verlauf der meisten psychischen Störungen ist variabel. Remissionen sind möglich, genauso wie Rückfalle und Langzeitbehinderungen. Die wissenschaftliche Forschung kämpft mit Fragen der Definition von Verläufen und methodischen Problemen in Langzeitstudien. Die klinische Nützlichkeit von prognostischen Indikatoren für individuelle PatientInnen ist begrenzt. Aus Angst vor enttäuschten Hoffnungen die Prognose eher negativ zu halten, ist keine seriöse Lösung dieser Probleme.

Argumente für die kürzlich prominent vorgeschlagenen Remissionskriterien für Schizophrenie beinhalten nicht nur Daten, die der Annahme eines notwendigerweise chronischen Verlaufs widersprechen, sondern berufen sich auch auf gute Erfahrungen mit der Einführung von Remissionskriterien für affektive und Angststörungen und stellen eine Erhöhung der Erwartungen sowie eine Intensivierung der therapeutischen Anstrengungen in Aussicht [3]. Das ist sicherlich notwendig, wenn man bedenkt, dass nur ein kleiner Teil aller PatientInnen tatsächlich Zugang zu allen Interventionen haben, von denen wissenschaftlich erwiesen ist, dass sie wirksam sind, und viele darunter leiden, dass sie zu wenig Un- 
terstützung für individuelle Behandlungsentscheidungen erhalten, sodass ein nicht geringer Teil ihrer Anstrengungen zur Überwindung der Störung und in Richtung Gesundheit außerhalb der therapeutischen Beziehung stattfindet.

Aus der Recovery-Forschung wissen wir auch, dass Hoffnung eine wesentliche Voraussetzung für Veränderungen in Richtung Gesundheit ist [4]. In Zeiten, in denen unser Wissen um Resilienzentwicklungen auch im Erwachsenenleben stark zunimmt und in denen die Erkenntnisse der Gesundheitsförderung sowie die Expertise für primäre, aber vor allem sekundäre und tertiäre Prävention immer wichtiger werden, sollte demoralisierender Pessimismus von rationalem Optimismus [5] abgelöst werden: Integrative multidimensionale Konzepte psychiatrischer Behandlung sollten ihre Basis finden in einer positiven Haltung und in einer Reduktion der vorherrschenden Skepsis gegenüber der Möglichkeit der Gesundung [6].

\section{Eine Diagnose einer psychiatrischen Erkrankung hindert einen nicht daran, Resilienz zu entwickeln. Wenn man sich jedoch zu vollständig mit der Krankheit und deren implizierten Beschrän- kungen identifiziert, kann dies einen davon abhalten, den nega- tiven Erfahrungen und Gefühlen sinnvolle eigene Reaktionen und Antworten entgegenzusetzen [7]}

Die Erfahrungen von Menschen, die selbst „in Recovery“ leben, haben für die Identifizierung von Genesungspotenzial und Möglichkeiten zur Selbstbestimmung und Eigeninitiative auch in Menschen, die sich in als aussichtslos erscheinenden Situationen befinden, große Bedeutung [8]. Sie können dem „bias“ der KlinikerInnen, die in ihrer Einschätzung auf klinische Erfahrungen mit Risiken, Krisen und Krankheit begrenzt sind, ihre Erfahrungen mit erfolgreichen Aufbrüchen aus solchen Situationen entgegensetzen ohne die Mühen einer über lange Zeit bestehenden Leidenssituation zu unterschätzen. Ihr Blick für zu stärkende Tendenzen zur Gesundheit auch in Phasen der Demoralisierung kann als peer support für Betroffene aber auch als Input in Betreuungsteams die Dynamik von Enttäuschung und Resignation verändern. Recovery-orientierte Einrichtungen arbeiten daher mit Betroffenen mit Recovery-Erfahrung zusammen, eine Entwicklung, die von vielen professionellen HelferInnen optimistisch eingeschätzt wird und im Kampf gegen Stigma und Diskriminierung eine entscheidende Rolle spielen könnte [9].

Die Begriffe „Schizophrenie“ und „chronische Erkrankung“ sind vom Mythos der Unheilbarkeit umgeben - unabhängig davon, was ihre medizinische Bedeutung ist. „Schizophrenie“ wird es möglicherweise als diagnostischen Begriff bald nicht mehr geben. In Großbritannien gibt es derzeit eine kritische Diskussion um das medizinische Modell der „chronischen Erkrankung“, das neben Diabetes und Herzerkrankungen auch Depression und Angststörungen zusammenfasst und das durch ein „kollaboratives Recovery-Modell“ für psychische Störungen ersetzt werden könnte [10].

Selbstbestimmung und Wahlfreiheit im Hinblick auf Behandlungsangebote, Orientierung an individuellen Lebenszielen und Ressourcen ebenso wie Hoffnung, Gesundheits- und Resilienzförderung sind wesentliche Kriterien recovery-orientierter Praxis. Das Recovery-Modell und seine Expertise für den persönlichen und gesellschaftlichen Kontext von Störung, Behinderung und Genesung könnte besser geeignet sein als das Modell der „chronischen Krankheit“, um die Anstrengungen von PatientInnen, ihren Familien und Freunden sowie der unterschiedlichen Gruppen professioneller HelferInnen optimal zu bündeln.

\section{Kontra}

Die Behandlungsstrategien in der Psychiatrie haben sich in den vergangenen Jahrzehnten dramatisch gewandelt und mit ihnen die Philosophie psychiatrischer Rahmenkonzepte. Präventive und multidisziplinäre Behandlungsmodelle gehören nun weitenteils zum Standard. Seit einiger Zeit werden nun auch vermehrt recoveryorientierte Behandlungsmodelle vorgestellt, als Gegengewicht oder gar zur Ablösung der Vorstellung von Chronizität beispielsweise schizophrener Erkrankungen. Recovery-orientierte Behandlungsmodelle sind hierbei auf die Wiederherstellung von „Normalität“ ausgerichtet. Die Schizophrenie wird in diesem Kontext als lediglich temporäre Phase im Leben eines Erkrankten angesehen, die durch entsprechende Unterstützungs- und Behandlungsangebote überwunden werden kann und nach welcher der Betroffene dann wieder ein „normales“ Leben wie vor seiner Erkrankung führen kann. Im Unterschied zum Chronizitätskonzept, das bei der Schizophrenie von einer in den meisten Fällen lebenslangen, chronischen Erkrankung ausgeht, bietet die Idee von Recovery mehr Raum für Optimismus und Hoffnung, was zunächst als durchaus positiv zu bewerten ist. Hoffnung zu vermitteln ist eine basale Aufgabe ärztlichen und psychiatrischen Handelns. Die vermittelte Hoffnung sollte jedoch auf realistischen Grundannahmen beruhen, da sie sonst schnell enttäuscht und in therapeutisch kontraproduktive Entwicklungen umschlagen kann. Die Befürworter recovery-orientierter Ansätze vermitteln, dass Ziele der Behandlung die stabile Remission der Symptomatik, ein unabhängiges Leben, die Rückkehr in den Arbeitsprozess und stabile partnerschaftliche und zwischenmenschliche Beziehungen etc. sind [11].

Diese Definition erinnert an die zu Recht vielfach kritisierte Definition von Gesundheit der WHO. Und angesichts allgemein hoher Arbeitslosenzahlen und über 50\% Single-Haushalten in Großstädten fragt man sich unwillkürlich, wie viele psychisch gesunde Menschen diese Recovery-Kriterien wohl erfüllen mögen? Für die Mehrheit schizophren erkrankter Menschen erscheinen diese Kriterien unrealistisch hochgesteckt und eher dazu geeignet eine unerfüllbare Aufgabe als eine positive Herausforderung zu sein, wodurch das Gegenteil dessen, was ursprünglich intendiert war, erreicht wird.

Erwähnt werden muss in diesem Zusammenhang jedoch fairerweise, dass die zuvor umrissene Definition von Recovery lediglich eine unter vielen, recht unterschiedlichen Definitionen darstellt, was aber nur auf ein weiteres Problem hinweist: Die Unklarkeit und Problematik dessen nämlich, was unter Recovery eigentlich zu verstehen ist. Recovery bedeutet im Wortsinn die Wiederherstellung eines Zustandes vor Eintritt eines bestimmten Ereignisses. Schon dies erscheint unrealistisch, da das Erleben einer Psychose und der damit verbundenen Ereignisse bereits für sich genommen Veränderungen bei dem Betroffenen, dessen Leben und der Wahrnehmung seiner durch andere bedingt, die eine Recovery im zuvor genannten Wortsinn verunmöglichen. Eine Recovery in diesem Sinne mag für einen Computer sinnvoll und möglich erscheinen, bei Menschen geht es jedoch eher darum, das einmal Erlebte sinnvoll in das weitere Leben $\mathrm{zu}$ integrieren und auch zu lernen, dass das Leben trotz chronischer Erkrankung sinnvoll und erfüllend sein kann. Dieser Lernprozess kann freilich nur dann nachhaltig erfolgreich gemeistert werden, wenn der Charakter der Erkrankung nicht negiert und die avisierten Therapieziele realistisch gehalten werden. Vom Optimismus der Mission der Recovery getragen suggerieren nun manche Autoren weiter, dass der Verlauf der Schizophrenie in der Regel überhaupt nicht chronisch sei und $50 \%$ oder 
gar mehr Patienten mit Schizophrenie günstige Verläufe aufweisen (z.B. [10]). Hierbei wird die Evidenzlage zum Verlauf der Schizophrenie allerdings sehr selektiv, um nicht zu sagen verzerrt dargestellt. Ausgeblendet werden von diesen Autoren beispielsweise Metaanalysen, die für schizophrene Erkrankungen Recovery-Raten von deutlich unter $40 \%$ [12] oder gar lediglich von um die 20\% („complete Recovery“, [13]) berichten. Bei Krankheitsverläufen von über zwei Jahren wurden in einer neueren Übersicht zu First-episode-Studien günstige Verläufe in nur $25 \%$ der Betroffenen gefunden [14]. Robinson et al. [15] fanden, dass in ihrer Verlaufsstudie zu ersterkrankten schizophrenen und schizoaffektiven Erkrankungen lediglich 13,7\% der Patienten im 5-Jahres-Zeitraum eine „full recovery“ erreichten (Symptomremission und adäquates soziales Funktionsniveau für einen Zeitraum von mindestens 2 Jahren).

Vergessen wird bei der Propagierung der Recovery und Infragestellung des Chronizitätskonzeptes gelegentlich auch, dass zahlreiche Leistungen der Gesundheits- und Sozialsysteme nur bei chronischen Erkrankungen erbracht werden. Die prinzipielle Infragestellung des Chronizitätskonzeptes bei der Schizophrenie kann insofern auch dazu führen, dass Ressourcen aus der Versorgung chronisch Kranker abgezogen werden und die Hürde für bestimmte Leistungen immer höher gelegt wird, was Ansporn, für viele Betroffene aber auch Überforderung bedeuten kann. Schon heute wird die Verteilung der Ausgaben dem Bedarf in der Basisversorgung nicht gerecht und insbesondere chronisch psychisch Kranke werden benachteiligt [16]. Diese Situation verschärft sich weiter, wenn mehr und mehr Ressourcen in lediglich für hochselektierte Patientengruppen zugängliche, öffentlichkeitswirksam darstellbare recovery-orientierte Versorgungsangebote fließen, deren Wirksamkeit im Hinblick auf eine nachhaltige Verbesserung der Krankheitsverläufe, nebenbei bemerkt, empirisch kaum ausreichend belegt ist.

Die Polarisierung von recovery-orientierter Behandlung als quasi „bessere“ oder „modernere“ Psychiatrie da und dem Chronizitätskonzept folgender Behandlung als „schlechtere“ oder „überkommene“ Psychiatrie dort erscheint nicht nur aufgrund der Evidenzlage, sondern und auch deswegen artifiziell, da natürlich auch unter der Vorstellung der Chronizität schizophrener Erkrankungen prinzipiell angestrebt wird, dem Patienten ein weitgehend normales Leben zu ermöglichen. Andererseits geht die Vorstellung von Chronizität möglicherweise allzu häufig, im Sinne einer Self-Fulfilling Prophecy, mit Pessimismus bezüglich der Behandlungsmöglichkeiten und langfristig erreichbaren Ziele einher, sodass ungünstige Verlaufsentwicklungen vielleicht zu schnell akzeptiert und als zu erwartende Konsequenz einer chronischen Erkrankung hingenommen werden, ohne dass zuvor bereits sämtliche zur Verfügung stehenden Behandlungs- und Unterstützungsoptionen ausgeschöpft wurden. Diesem dem Chronizitätskonzept sicherlich innewohnenden Pessimismus und Fatalismus muss entgegengesteuert werden und hier leistet die Idee von Recovery zweifelsohne einen wertvollen Beitrag. Dies bedeutet allerdings nicht, dass deswegen das Konzept von der Chronizität der Schizophrenie, das ja empirisch hinreichend belegt ist, aufzugeben ist. Das Chronizitätskonzept sollte vielmehr durch andere Konzepte beispielsweise das der Recovery sinnvoll gegengewichtet und ergänzt werden. Eine Polarisierung beider Konzepte in dem weiter oben genannten Sinne kann dabei nur abträglich sein.

\section{Literatur}

1 Greve N. Was lange währt ... wird nicht immer gut: Risiken der chronischen Betreuung. Soziale Psychiatrie 2007; 118: 15 - 17

2 Ludwig AM, Farrelly F. The code of chronicity. Arch Gen Psychiatry 1966; 15: $562-568$

3 Andreasen NC, Carpenter WT, Kane JM, Lasser RA, Marder SR, Weinberger DR. Remission in schizophrenia: proposed criteria and rationale for consensus. American Journal of Psychiatry 2005; 162: 441 - 449

4 Amering M, Schmolke M. Recovery. Das Ende der Unheilbarkeit. Bonn: Psychiatrie-Verlag, 2007

$5 \mathrm{Knuf}$ A. Vom demoralisierenden Pessimismus zum vernünftigen Optimismus. Eine Annäherung an das Recovery-Konzept. Soziale Psychiatrie 2004; $1: 38-41$

6 WPA. www.wpanet.org 2005

7 Glover H. www.mentalhealth.org.uk 2003

8 Amering M. Recovery - warum nicht? Psychiat Prax 2008; 35 (2): 55 56

9 Sibitz I, Swoboda H, Schrank B, Priebe S, Amering M. Einbeziehung von Betroffenen in Therapie- und Versorgungsentscheidungen: professionelle HelferInnen zeigen sich optimistisch. Psychiat Prax 2008; 35: $128-134$

10 Lester H, Gask L. Delivering medical care for patients with serious mental illness or promoting a collaborative model of recovery? British Journal of Psychiatry 2006; 188: $401-402$

11 Liberman RP, Kopelowicz A, Ventura J, Gutkind D. Operational criteria and factors related to recovery from schizophrenia. International Review of Psychiatry 2002; 14: 256- 272

12 Hegarty JD, Baldessarini RJ, Tohen M, Waternaux C, Oepen G. One hundred years of schizophrenia: a meta-analysis of the outcome literature. Am J Psychiatry 1994; 151 (10): 1409-1416

13 Warner R. Recovery from Schizophrenia: Psychiatry and Political Economy (3rd edn). London: Brunner-Routledge, 2003

14 Menezes NM, Arenovich T, Zipursky RB. A systematic review of longitudinal outcome studies of first-episode psychosis. Psychol Med 2006; 36 (10): $1349-1362$

15 Robinson DG, Woerner MG, McMeniman M, Mendelowitz A, Bilder RM. Symptomatic and functional recovery from a first episode of schizophrenia or schizoaffective disorder. Am J Psychiatry 2004; 161 (3): $473-479$

16 Melchinger $H$, Rössler W, Machleidt W. Ausgaben in der psychiatrischen Versorgung: Ist die Verteilung der Ressourcen am Bedarf orientiert? Nervenarzt 2006; 77 (1): $73-80$ 